\title{
Die Koexistenz der Zugewinn- und der Errungenschaftsgemeinschaft
}

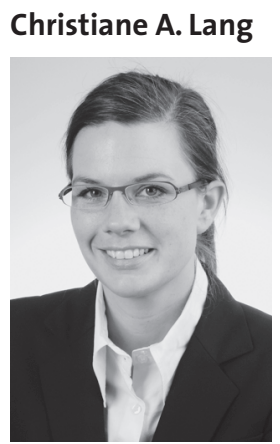

Mitglied der Kommission Zivil-, Familienund Erbrecht, Recht anderer Lebensgemeinschaften im djb, Rechtsanwältin, Berlin
Im Zuge der Auseinandersetzung mit dem am 2. November 2007 durch das Bundesministerium der Justiz versandten Entwurf eines Gesetzes zur Änderung des Zugewinnausgleichsrechts rückt die „gute alte“ Zugewinngemeinschaft in der Bundesrepublik Deutschland in den Mittelpunkt der Diskussion. Insbesondere nach einem Blick zu unseren europäischen Nachbarn könnte die vermögensrechtliche Alternative des gesetzlichen Güterstands der Errungenschaftsgemeinschaft in Betracht gezogen und die „Existenzberechtigung“ der Zugewinngemeinschaft in Frage gestellt werden. Denn Deutschland ist eines der wenigen Länder, in denen die Zugewinngemeinschaft den gesetzlichen Güterstand darstellt; im europäischen Ausland dominiert indes der Güterstand der Errungenschaftsgemeinschaft.

\section{Zugewinngemeinschaft}

Schließen in der Bundesrepublik Deutschland zwei Personen die Ehe miteinander und treffen keine abweichende vertragliche Regelung im Sinne des $\mathbb{S}$ 1408 Abs. 1 BGB, leben sie im gesetzlichen Güterstand der Zugewinngemeinschaft.

Der Gesetzgeber sieht in $\$ 1363$ Abs. 2 S. 1 BGB ausdrücklich vor, dass das Vermögen des Mannes und das Vermögen der Frau nicht gemeinschaftliches Vermögen werden. Diese Vermögenstrennung gilt auch für das nach Eheschließung und damit während der Ehe erworbene Vermögen.

Im Sinne des $\mathbb{} 1363$ Abs. 2 S. 2 BGB wird nur der Zugewinn - gemäß der Legaldefinition des $\mathbb{S}$ 1373 BGB der Betrag, um den das Endvermögen eines Ehegatten das Anfangsvermögen übersteigt ausgeglichen. Dem Zugewinnausgleich liegt ein gesondert für jeden Ehegatten durchzuführender Vergleich des Anfangsvermögens zur Zeit der Eheschließung mit dem Endvermögen zum Ende des Güterstands zugrunde. Ergibt sich eine positive Differenz, stellt diese den jeweiligen (Zu-)Gewinn des einzelnen Ehegatten dar. Übersteigt der Zugewinn des einen den des anderen Ehegatten, ist derjenige mit dem höheren Zugewinn in Höhe der Hälfte der Differenz ausgleichspflichtig.

Dieser Grundsatz der getrennten Vermögenszuteilung und auch Vermögensmehrung soll - unberührt von den Reformbestrebungen - auch weiterhin bestehen bleiben.

\section{Errungenschaftsgemeinschaft}

Eine andere Güterstandsform ist die der Errungenschaftsgemeinschaft. Die Errungenschaftsgemein- schaft ist eine Form der Gütergemeinschaft, in der das von den Eheleuten in der Ehe erworbene bzw. errungene Gut gemeinschaftliches Vermögen wird, die vorehelichen Vermögen dagegen getrenntes Vermögen des jeweiligen Inhabers bleiben. ${ }^{1}$

Die Gemeinsamkeit der Zugewinn- und Errungenschaftsgemeinschaft liegt somit darin, dass in der Zeit vor der Ehe (jedenfalls grundsätzlich) jeder Ehegatte Vermögen in Alleineigentum erwirbt.

Der Unterschied der beiden Güterstände liegt darin, dass das während der Ehe erworbene Vermögen bei der Errungenschaftsgemeinschaft gemeinschaftliches Vermögen und automatisch Miteigentum der Eheleute wird, bei der Zugewinngemeinschaft hingegen eine Trennung der Vermögensmassen gilt und mit dem Erwerbsvorgang nicht eheliches Gesamtgut begründet wird.

\section{Die deutsche Koexistenz beider Güterstände}

Ohne im Folgenden auf die einzelnen Beweggründe für die Wahl des gesetzlichen Güterstands der Errungenschaftsgemeinschaft unserer europäischen Nachbarn näher einzugehen und die vermeintlichen Vorzüge einer der beiden Güterstände darzustellen, soll im Rahmen dieser Ausführungen allein die deutsche Besonderheit hervorgehoben werden. Denn in der Bundesrepublik Deutschland bestehen die Zugewinngemeinschaft und die Errungenschaftsgemeinschaft in Koexistenz nebeneinander.

Das Bürgerliche Gesetzbuch ließ zunächst die Errungenschaftsgemeinschaft als vertraglichen Güterstand zu. Durch das Gleichberechtigungsgesetz vom 18. Juni 1957 wurde sie beseitigt und die Zugewinngemeinschaft ist seit dem Inkrafttreten dieses Gleichberechtigungsgesetzes am 1. Juli 1958 in den alten Bundesländern der gesetzliche Güterstand.

Seit der deutschen Wiedervereinigung und nach dem Inkrafttreten des BGB im Beitrittsgebiet am 3. Oktober 1990 gilt auch dort die Zugewinngemeinschaft. Der gesetzliche Güterstand des Familiengesetzbuchs der DDR (FGB/DDR), die Errungenschaftsgemeinschaft in Form der sogenannten Eigentums- und Vermögensgemeinschaft der Ehegatten, ging grundsätzlich von Gesetzes wegen in den neuen Güterstand der Zugewinngemeinschaft über. Anderes galt nur, wenn die Eheleute einer zu DDRZeiten geschlossenen Ehe von der ihnen in der

1 So etwa die Definition bei Köbler, Juristisches Wörterbuch, 11. Aufl., 2002; Deutsches Rechts-Lexikon, Bd. 1, 2. Aufl., 1992. 
Übergangsvorschrift des Art. $234 \mathbb{\$} 4$ Abs. 2 S. 1 EGBGB eröffneten Möglichkeit Gebrauch machten, bis zum Ablauf von zwei Kalenderjahren nach dem Beitritt und damit bis zum 2. Oktober 1992 die Option für die Fortgeltung des alten DDRGüterstands auszuüben und zuvor keinen Ehevertrag geschlossen hatten. ${ }^{2}$ In diesen Fällen wurde der bisherige Güterstand nach dem FGB/DDR auch nach der Wiedervereinigung beibehalten (Art. $234 \mathbb{S} 4$ Abs. 1 EGBGB) ${ }^{3}$ und die Regeln der Errungenschaftsgemeinschaft nach dem FGB/DDR gelten über den 3. Oktober 1990 weiter fort.

Auch für die „Güterstandswechsel-Ehen“ jedoch folgt aus dem Inkrafttreten des BGB keineswegs die Bedeutungslosigkeit des Güterrechts nach dem FGB/DDR, da die durch die Wiedervereinigung bedingte Änderung des gesetzlichen Güterstands erst mit Wirkung ex nunc eintrat. Für die eheliche Auseinandersetzung des bis zur Wirksamkeit des Beitritts erworbenen Vermögens haben daher die Vorschriften des FGB/ DDR weiter Bestand - für alle zu Zeiten der DDR geschlossenen Ehen. ${ }^{4}$

Im Scheidungsfall ist somit das zu DDR-Zeiten erworbene Vermögen nach dem Familiengesetzbuch der DDR - namentlich der $\mathbb{S} 39,40$ FGB/DDR - und das nach der Wiedervereinigung erwirtschaftete Vermögen der „GüterstandswechselEhen " nach den Regeln der Zugewinngemeinschaft nach dem BGB auseinanderzusetzen. ${ }^{5}$

\section{Stichtagsproblematik}

Ist das Vermögen einer zu DDR-Zeiten geschlossenen Ehe sowohl nach dem FGB/DDR als auch nach dem BGB auseinanderzusetzen, hat dies Auswirkungen auf die ursprünglich nach beiden Gesetzen vorgesehenen Stichtage, d.h. die konkreten Kalendertage, die für die Wertbestimmung der auseinanderzusetzenden Vermögensgegenstände entscheidend sind. Da „Beginn“ und „Ende“ der Ehe im jeweils anderen gesetzlichen Geltungsbereich liegen - namentlich die Eheschließung im gesetzlichen Geltungsbereich des FGB/DDR und das Güterstandsende im gesetzlichen Geltungsbereich des BGB -, sind die Stichtage zeitlich zu modifizieren.

Bei der Anwendung der Regelungen des FGB/DDR sind für die Ermittlung der Vermögenswerte bei Beendigung des Güterstands nicht die Verhältnisse bei Zustellung des Scheidungsantrags ${ }^{6}$, sondern die Verhältnisse bei Beendigung des DDRGüterstands durch den Übergang in die Zugewinngemeinschaft und damit die Verhältnisse am 3. Oktober 1990 maßgebend. ${ }^{7}$ Für die Zugewinngemeinschaft gilt, dass der Stichtag für das Anfangsvermögen nicht der noch in die Zeit des Bestehens der DDR fallende Tag der Eheschließung, sondern der Tag des 3. Oktober 1990 ist, an dem die Zugewinngemeinschaft mit dem wirksamen Beitritt gesetzlicher Güterstand wurde. ${ }^{8}$ Bei der vermögensrechtlichen Auseinandersetzung ist somit der Tag der Wiedervereinigung am 3. Oktober 1990 in der Praxis der „doppelte“ bzw. zweifache Stichtag.

Stichtag für die Bestimmung des Endvermögens nach dem 3. Oktober 1990 bleibt im Sinne des BGB der Tag der Zustellung des Ehescheidungsantrags. ${ }^{9}$

\section{Vermögensrechtliche Auseinandersetzung nach dem FGB/DDR}

$\mathrm{Da}$ in jedem Scheidungsfall einer zu DDR-Zeiten geschlossenen Ehe das FGB/DDR zur Anwendung gelangt, werden nachstehend die wesentlichen Grundsätze der vermögensrechtlichen Auseinandersetzung nach den $\mathbb{S}$ 39, 40 FGB/DDR dar-

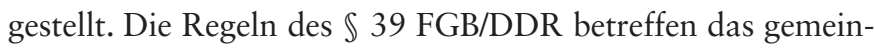
schaftliche, die des $\mathbb{4} 40 \mathrm{FGB} / \mathrm{DDR}$ das alleinige Vermögen eines Ehegatten.

Zunächst sind daher - wie auch im Rahmen des Zugewinnverfahrens - die Eigentumsverhältnisse der Eheleute an den jeweils auseinanderzusetzenden Vermögensgegenständen zu klären.

Die Begründung der Eigentums- und Vermögensverhältnisse der Ehegatten richtet sich nach den Regeln des $\mathbb{} 13$ FGB/ DDR. Gemäß $\ 13$ Abs. 1 S. 1 FGB/DDR gehören die von einem oder beiden Ehegatten während der Ehe durch Arbeit oder aus Arbeitseinkünften erworbenen Sachen, Vermögensrechte und Ersparnisse beiden Ehegatten gemeinsam. Das gemeinschaftliche Eigentum entsteht während der Ehe kraft Gesetzes und ist eine Form des Gesamteigentums ( $\mathbb{S} 34$ Abs. 2 i.V.m. $\int 42$ Abs. 3 ZGB). ${ }^{10}$

Gemäß $\int 13$ Abs. 2 FGB/DDR gehören jedem Ehegatten die vor Eheschließung erworbenen, die ihm während der Ehe als Geschenk oder als Auszeichnung zugewendeten und die durch Erbschaft zugefallenen Sachen und Vermögensrechte allein. Die Art des Erwerbs auf das vor der Eheschließung erreichte Vermögen ist auf dessen Zuordnung zum Alleineigentum ohne Einfluss. ${ }^{11}$

Eine Besonderheit sieht $\$ 299$ ZGB/DDR für den Erwerb von Grundstücken durch einen Ehegatten vor. Danach wird ein Grundstück stets gemeinschaftliches Eigentum der Ehegatten, wenn nicht eine abweichende Erklärung der Ehegatten abgegeben oder die eheliche Vermögensgemeinschaft bereits rechtskräftig aufgehoben ist. ${ }^{12}$

2 Götsche, FamRB 6/2003, 189, 190; Schael, NJ 7/2004, 289.

3 BGH v. 5.5.1999 - XII ZR 184/97, FamRZ 1999, 1197, 1198 = NJW 1999, 2520 ff.; Die Bedeutung für die familienrechtliche Praxis erlangte jedoch geringen Wert, da nur etwa 3.700 Ehepaare von ihrem Optionsrecht Gebrauch machten, vgl. Peters, Registerverfahrensbeschleunigungsgesetz, FamRZ 1994, 673, 674.

4 BGH v. 5.6.2002 - XII ZR 194/o0, FamRZ 2002, $1097=$ MDR 2002, $1068 \mathrm{f}$.

5 BGH v. 5.6.2002 - XII ZR 194/o0, FamRZ 2002, $1097=$ MDR 2002, 1068 f., in Fortführung von BGH v. 5·5.1999 - XII ZR 184/97, FamRZ 1999, 1197, 1198 = NJW 1999, $2520 \mathrm{ff}$.

6 Die Wertverhältnisse bei Eintritt der Rechtskraft der Ehescheidung sind nur dann entscheidend, wenn sich die vermögensrechtliche Auseinandersetzung ausschließlich nach den $\S \S 39,40$ FGB/DDR richtet und daneben kein Zugewinnausgleichsrecht anzuwenden ist.

7 BGH v. 5.5.1999 - XII ZR 184/97, FamRZ 1999, 1197, 1198 = NJW 1999, $2520 \mathrm{ff}$.

8 BGH v. 5.5.1999 - XII ZR 184/97, FamRZ 1999, 1197, 1198 = NJW 1999 , 2520 ff.; Götsche, Die Anwendung der $\$ \$ 39,40$ FGB/DDR bei der vermögensrechtlichen Auseinandersetzung im Scheidungsfall (IV), FamRB 10/2003, 339.

9 Palandt-Brudermüller, BGB, 63. Aufl. 2004, §1376 Rn. 4, §1384 Rn. 5.

10 FGB-Kommentar, Staatsverlag der DDR, 5. Aufl. 1982, § 13 Anm. 1.1 und 1.3.

11 FGB-Kommentar, Staatsverlag der DDR, 5. Aufl. 1982, § 13 Anm. 2.1.

12 OLG Brandenburg v. 6.11.2001 - 9 UF 39/01, OLGReport Brandenburg 2002, 516, 517; ZGB-Kommentar, Staatsverlag der DDR, 2. Aufl. 1985, \$299 Anm. 1.1. 


\section{Verteilung gemeinschaftlichen Eigentums nach § 39 FGB/DDR} \39 FGB/DDR regelt die Auseinandersetzung des während der Ehe errungenen gemeinschaftlichen Eigentums und ist auf Zuweisung der einzelnen Vermögenswerte gerichtet.

Bei Beendigung der Ehe wird das gemeinschaftliche Vermögen im Sinne des $\ 39$ Abs. 1 S. 1 FGB/DDR zu gleichen Anteilen auseinandergesetzt und geteilt. Es findet grundsätzlich eine Zuweisung der einzelnen Vermögensgegenstände jeweils zu Alleineigentum der Ehegatten statt. ${ }^{13}$ Von wesentlicher Bedeutung ist hierbei, dass die Teilung nach dem sogenannten Halbteilungsgrundsatz zu gleichen Teilen erfolgen soll. Ist eine Teilung in Natur möglich, so hat diese auch möglichst genau zu erfolgen. ${ }^{14}$ Ist eine Teilung in Natur nicht möglich, wird an dem unteilbaren Vermögensgegenstand hälftiges Miteigentum begründet.

Nur in Ausnahmefällen soll eine Zuteilung zu ungleichen Anteilen am gemeinschaftlichen Eigentum gemäß $\ 39$ Abs. 2 S. $1 \mathrm{FGB} / \mathrm{DDR}$ bis hin zur Übertragung von Alleineigentum führen. In diesen Fällen ungleicher Verteilung entsteht dann jedoch ein auf Geld gerichteter Erstattungsanspruch desjenigen, der den Vermögensgegenstand zum geringeren Anteil oder nicht zugeteilt erhält ( $\mathbb{S} 3$ Abs. 1 S. 3 FGB/DDR). ${ }^{15}$

\section{Verteilung alleinigen Eigentums nach $\S 40$ FGB/DDR}

\40 FGB/DDR regelt die Teilhabe eines Ehegatten am Alleineigentum des anderen Ehegatten. Anders als die Regelung des $\int 39 \mathrm{FGB} / \mathrm{DDR}$ ist keine Aufteilung des Alleineigentums zugunsten des anspruchsberechtigten Ehegatten vorgesehen. Der Ausgleichsanspruch des $\mathbb{4} 40 \mathrm{FGB} / \mathrm{DDR}$ ist von vornherein als ein Anspruch auf Geld konzipiert und dient dem Ausgleich für während der Ehe beigetragene Leistungen des einen Ehegatten zur Vergrößerung oder Erhaltung des Alleineigentums des anderen. ${ }^{16}$

Die Höhe des Ausgleichsanspruchs unterliegt grundsätzlich richterlichem Ermessen und ist unter Abwägung aller Umstände des Einzelfalls zu bestimmen. ${ }^{17}$ Die höchstzulässige Grenze des Anspruchs ist jedoch gesetzlich in $\mathbb{\int} 40$ Abs. 2 S. 1 FGB/ DDR festgesetzt. Der als Ausgleich zuzusprechende Anteil darf die Hälfte des vorhandenen Vermögens, zu dessen Mehrung oder Erhaltung der andere Ehegatte beigetragen hat, nicht übersteigen. ${ }^{18}$

Besonders hervorzuheben ist an dieser Stelle, dass sich der Ausgleichsanspruch nicht an dem durch die Beitragsleistungen eingetretenen Wertzuwachs orientiert, sondern $\mathbb{S} 40 \mathrm{FGB} /$ DDR einen Geldanspruch gewährt, dessen Umfang sich nach dem durch den Wertzuwachs erhöhten (End-)Wert des Vermögensgegenstandes richtet, zu dessen Mehrung oder Erhaltung der andere Ehegatte beigetragen hat. ${ }^{19}$

\40 Abs. 1 FGB/DDR setzt ferner einen wesentlichen Beitrag zur Vergrößerung oder Erhaltung des Vermögens voraus, wobei der Beitrag sowohl aus unmittelbarer Arbeitsleistung als auch aus mittelbarer Leistung wie Haushaltsführung und Kindererziehung bestehen kann. ${ }^{20}$

\section{Auskunftsanspruch aus $\$ 242$ BGB}

Dem nach den $\mathbb{S} 339,40$ FGB/DDR ausgleichsberechtigten Ehegatten steht gegenüber dem Verpflichteten ein Auskunftsanspruch über dessen Vermögenswerte aus $\mathbb{} 242 \mathrm{BGB} z u,{ }^{21}$ der analog der Stufenklage gemäß $\mathbb{2 5 4}$ ZPO i.V.m. $\ 260$ BGB gilt. ${ }^{22}$

Das FGB/DDR enthielt zwar keine ausdrückliche Regelung, die den einen Ehegatten zur klageweisen Einholung von Auskünften über das Vermögen des anderen berechtigte. Nach der Rechtsauffassung der ehemaligen DDR war dennoch die Klage auf Auskunftserteilung über das ausgleichspflichtige Vermögen möglich, wenn der Kläger seine Forderung aus Unkenntnis über den Wertumfang nicht konkret beziffern konnte, der Beklagte hingegen über die entsprechenden Informationen verfügte und diese verweigerte. ${ }^{23}$ Dies entspricht inhaltlich dem Auskunftsanspruch im Sinne des $\mathbb{2} 24$ BGB.

\section{Verfahrensrecht der ZPO}

Die Geltendmachung der Ansprüche aus $\mathbb{\$} \mathbb{S} 39,40 \mathrm{FGB} / \mathrm{DDR}$ richtet sich verfahrensrechtlich nach der ZPO. ${ }^{24}$ Es handelt sich um Familiensachen gemäß $\$ 621$ Abs. 1 Ziff. 8 ZPO 25 und erstinstanzlich sachlich zuständig sind die Familiengerichte der Amtsgerichte gemäß $\mathbb{S}$ 23a Ziff. 5, 23b Abs. 1 Ziff. 9 GVG. ${ }^{26}$

\section{Fazit}

Die Praxis der vermögensrechtlichen Auseinandersetzung nach dem FGB/DDR hat auch nach dem 3. Oktober 1990 seine Bedeutung nicht gänzlich verloren und die Koexistenz der Errungenschafts- neben der Zugewinngemeinschaft ist eine deutsche Besonderheit, die auch durch die aktuelle Reform des Güterrechts unberührt bestehen bleibt.

13 FGB-Kommentar, Staatsverlag der DDR, 5. Aufl. 1982, § 39 Anm. 1.5 und 1.6.5.

14 BGH v. 29.1.1992 - XII ZR 241/90, FamRZ 1992, 531, 532 f.

15 BGH v. 15.1.1992 - XII ZR 202/90, FamRZ 1992, 414, $415=$ NJW 1992, 821

16 S. auch BGH v. 5.5.1999 - XII ZR 184/97, FamRZ 1999, 1197,1198 = NJW 1999, 2520 ff.; vgl. \$ 40 Abs. 1, 2 FGB/DDR.

17 BGH v. 5.6.2002 - XII ZR 194/o0, FamRZ 2002, 1097, $1098=$ MDR 2002, $1068 \mathrm{f}$.

18 Eberhardt/Daute/Duft, Antwort auf erste Fragen zur Anwendung des Familiengesetzbuches, NJ 1966, 289, 290.

19 BGH v. 5.5.1993-XII ZR 38/92, FamRZ 1993, 1048, $1049=$ MDR 1993, $983 \mathrm{f}$.

20 Für eine vertiefte Darstellung der Anspruchsvoraussetzungen des $\S 40$ FGB/DDR s. auch Lang, FORUM Familien- und Erbrecht 2005, Heft 1/2, 29 ff.

21 BGH v. 5.5.1999 - XII ZR 184/97, FamRZ 1999, 1197, $1199=$ NJW 1999 , $2520 \mathrm{ff}$.

22 Palandt-Brudermüller, BGB, 63. Aufl. 2004, Art. 234 § 4 EGBGB Rn. 16.

23 Lehrbuch Familienrecht, Staatsverlag der DDR, 8. Aufl. 1989, Kap. V, 191.

24 BGH v. 5.5.1993 - XII ZR 38/92, FamRZ 1993, 1048, $1049=$ MDR 1993, 983 f.; so auch KG v. 30.1.1992 - 16 UF 5325/91, FamRZ 1992, 566, $567=$ NJ 1992, 217 f.; a.A. Buschhaus, Die Auseinandersetzung der Eigentums- und Vermögensgemeinschaft, Diss. Göttingen 1999, 218 ff., 240.

25 BGH v. 5.5.1993 - XII ZR 38/92, FamRZ 1993, 1048, $1049=$ MDR 1993 983 f.; so bereits BGH (Beschluss) v. 20.3.1991 - XII ZR 202/90, FamRZ 1991, 794, $795=$ MDR 1991, 789 f

26 Thomas/Putzo-Hüßtege, ZPO, 26. Aufl. 2004, § 23b GVG Rn. 3. 